ARTICLE

Received 8 Oct 2013 | Accepted 30 Apr 2014 | Published 2 Jun $2014 \quad$ DOl: 10.1038/ncomms5011

\title{
De novo SOX11 mutations cause Coffin-Siris syndrome
}

Yoshinori Tsurusaki ${ }^{1, \star}$, Eriko Koshimizu ${ }^{1, \star}$, Hirofumi Ohashi ${ }^{2}$, Shubha Phadke ${ }^{3}$, Ikuyo Kou ${ }^{4}$, Masaaki Shiina ${ }^{5}$, Toshifumi Suzuki ${ }^{1,6}$, Nobuhiko Okamoto ${ }^{7}$, Shintaro Imamura ${ }^{8}$, Michiaki Yamashita ${ }^{8}$, Satoshi Watanabe ${ }^{9}$, Koh-ichiro Yoshiura9', Hirofumi Kodera', Satoko Miyatake1, Mitsuko Nakashima', Hirotomo Saitsu', Kazuhiro Ogata ${ }^{5}$, Shiro Ikegawa ${ }^{4}$, Noriko Miyake ${ }^{1} \&$ Naomichi Matsumoto ${ }^{1}$

Coffin-Siris syndrome (CSS) is a congenital disorder characterized by growth deficiency, intellectual disability, microcephaly, characteristic facial features and hypoplastic nails of the fifth fingers and/or toes. We previously identified mutations in five genes encoding subunits of the BAF complex, in $55 \%$ of CSS patients. Here we perform whole-exome sequencing in additional CSS patients, identifying de novo SOX11 mutations in two patients with a mild CSS phenotype. sox11a/b knockdown in zebrafish causes brain abnormalities, potentially explaining the brain phenotype of CSS. SOX11 is the downstream transcriptional factor of the PAX6-BAF complex, highlighting the importance of the BAF complex and SOX11 transcriptional network in brain development.

\footnotetext{
${ }^{1}$ Department of Human Genetics, Yokohama City University Graduate School of Medicine, 3-9 Fukuura, Kanazawa-ku, Yokohama 236-0004, Japan.

${ }^{2}$ Division of Medical Genetics, Saitama Children's Medical Center, 2100 Magome, Iwatsuki 339-8551, Japan. ${ }^{3}$ Department of Medical Genetics, Sanjay Gandhi Postgraduate Institute of Medical Sciences, Raebareli Rd, Lucknow 226014, India. ${ }^{4}$ Laboratory for Bone and Joint Diseases, Center for Integrative Medical Sciences, RIKEN, 4-6-1 Shirokanedai, Minato-ku, Tokyo 108-8639, Japan. ${ }^{5}$ Department of Biochemistry, Yokohama City University Graduate School of Medicine, 3-9 Fukuura, Kanazawa-ku, Yokohama 236-0004, Japan. ${ }^{6}$ Department of Obstetrics and Gynecology, Juntendo University, Hongo 3-1-3, Bunkyo-ku, Tokyo 113-8431, Japan. ${ }^{7}$ Department of Medical Genetics, Osaka Medical Center and Research Institute for Maternal and Child Health, 840 Murodo-cho, Izumi 594-1101, Japan. ${ }^{8}$ National Research Institute of Fisheries Science, 2-12-4 Fukuura, Kanazawa-ku, Yokohama 236-8648, Japan. ${ }^{9}$ Department of Human Genetics, Nagasaki University Graduate School of Biomedical Sciences, 1-12-4 Sakamoto, Nagasaki 852-8523, Japan. ${ }^{\star}$ These authors contributed equally to this work. Correspondence and requests for materials should be addressed to N.M. (email: naomat@yokohama-cu.ac.jp).
} 
C offin-Siris syndrome (CSS; MIM\#135900) is a congenital disorder characterized by growth deficiency, intellectual disability, microcephaly, characteristic facial features and hypoplastic nails of the fifth fingers and/or toes (Supplementary Fig. 1). Five subunit genes (SMARCB1, SMARCA4, SMARCE1, $A R I D 1 A$ and $A R I D 1 B$ ) of the BAF complex (also known in yeast as the SWI/SNF complex ${ }^{1}$ ) are mutated in $55-70 \%$ of CSS patients $^{2-6}$. Mutations in SMARCA2, another BAF complex gene, were reported in the Nicolaides-Baraitser syndrome, which is similar to, but distinct from $\mathrm{CSS}^{7}$. Furthermore, de novo PHF6 mutations were found in two CSS patients ${ }^{6}$, although no direct interaction has been reported between the BAF complex and PHF6, which interacts with the nucleosome remodelling and deacetylation complex ${ }^{6}$. As $30-45 \%$ of CSS patients were genetically undiagnosed in three large cohort studies ${ }^{2-6}$, further genetic investigation is required to fully address the genetic picture of CSS.

Here we apply whole-exome sequencing (WES) to 92 CSS patients, and identify two de novo SOX11 mutations in two unrelated patients. sox11 knockdown experiments in zebrafish result in a smaller head and significant mortality, which were partially rescued by human wild-type SOX11 messenger RNA (mRNA), but not by mutant mRNA.

\section{Results}

WES of CSS patients. We identified two de novo SOX11 mutations in two unrelated patients, c.347A $>\mathrm{G}$ (p.Tyr116Cys) (in patient 1) and c.178T $>C$ (p.Ser60Pro) (in patient 2) (deposited to LOVD, http://www.LOVD.nl/SOX11), among 92 CSS patients (including our previous cohorts ${ }^{2,3}$ ) analysed by trio-based WES. In the two patients, $>10$ reads covered $94-92 \%$ of coding sequences and only SOX11 mutations remained as candidate variants in both of them based on the de novo model with scores of damaging or disease causing by SIFT, PolyPhen 2 and Mutation Taster (Supplementary Table 1). The two heterozygous mutations localize to the high-mobility group (HMG) domain. Neither mutation was registered in the databases examined $(1,000$ Genomes, Exome Sequencing project (ESP) 6500, and in-house databases containing 575 control exomes) (Supplementary Table 1). We identified a further 22 SOX11 variants within these three databases, but all of them reside outside the HMG domain and, based on prediction programs, are less likely to be pathogenic (Supplementary Table 1; Supplementary Fig. 2). The amino acids altered in SOX11 are evolutionarily conserved from zebrafish to human (Fig. 1). The mutations do not alter nuclear localization of SOX11 protein (Supplementary Fig. 3). De novo mutations were confirmed in the two families by Sanger sequencing along with biological parentage. No mutations in any of the other BAF complex genes, PHF6, or other potential

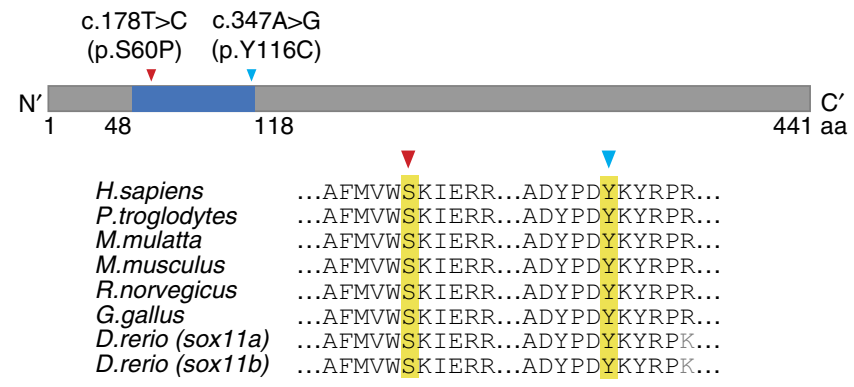

Figure 1 | SOX11 mutations and functional characterization. SOX11 mutations in CSS patients. Two missense mutations in the HMG domain (blue box) occur at evolutionarily conserved amino acids. candidate genes were found in the two families. Therefore, the two mutations identified are highly likely to be pathogenic. Moreover, SOX11 was sequenced by WES $(n=23)$ or Sanger method $(n=67)$ in a further 90 CSS patients, with no mutations found. Fifty-four patients had a mutation in one of the five BAF complex subunit genes (58.7\%) (SMARCA4, SMARCB1, SMARCE1, ARID1A and ARID1B mutations found in $9,8,1,5$ and 31 patients, respectively).

Clinical features of patients with SOX11 mutations. The two patients showed dysmorphic facial features, microcephaly, growth deficiency, hypoplastic fifth toe nails and mild intellectual disability $^{8}$ (Supplementary Fig. 1; Supplementary Table 2). The observed clinical features in both patients are classified to a mild end of CSS as patient 1 spoke early for CSS and patient 2 has relatively high intelligence quotient. Although the two patients do not look similar in facial appearance (patient 1 has midface hypoplasia, while patient 2 does not; in addition there is an ethnic difference, as patients were either Japanese or Indian), they do share features in common, namely, hypertrichosis, arched eyebrows, low-set ears, auricular back-rotationand full cheeks (Supplementary Fig. 1).

Patient 1 (Japanese) was born at 38 weeks of gestation following an uneventful pregnancy. Her birth weight was $2,340 \mathrm{~g}$ ( -1.9 s.d.), length $45 \mathrm{~cm}(-2.2$ s.d.) and occipitofrontal circumference (OFC) $30.5 \mathrm{~cm}$ (-1.8 s.d.). She was hypotonic, had feeding difficulties (especially during the neonatal period) and delayed development. She was able to support her head at 5 months of age, sit at 11 months and walk independently at 1 year 11 months. She started to speak meaningful words at 1 year 7 months. At 3 years, her developmental quotient was estimated using the Kyoto scale of psychological development to be 57 . Abdominal echography showed her left kidney was slightly small in size. She has distinctive facial features characterized by midface hypoplasia, short palpebral fissures, hypertelorism, upturned palpebral fissures, long eyelashes, a low nasal root, shortened nose with upturned nostrils, short philtrum, open mouth, full lips and low-set ears. Hypoplastic distal phalanges with nail hypoplasia (especially of the fifth digits) were also noted. Additional findings included hypertrichosis and long eyelashes with abundant hair on the scalp. At 4 years 8 months, she was short with a height of $92.1 \mathrm{~cm}$ ( -2.9 s.d.) and evaluated for possible growth hormone deficiency with stimulation tests, which showed normal results. At 10 years, she measured $119 \mathrm{~cm}(-2.8$ s.d.), weighed $20.1 \mathrm{~kg}$ ( -1.8 s.d.) and had an OFC of $47.3 \mathrm{~cm}(-3.3$ s.d.). She attends a special education class for poor performance, but can walk to school by herself (takes approximately half an hour) and is able to communicate verbally, to some extent, with her classmates. Clinical features are summarized in Supplementary Table 2.

Patient 2 (Indian) is a 16-year-old female, and was referred to the genetics outpatient department for evaluation of short stature. She was born at term following a normal pregnancy, but with low birth weight $(1.75 \mathrm{~kg},-4 \mathrm{~s} . \mathrm{d}$.). Developmental milestones were attained normally, but her parents always felt that she lagged behind other children. She was a slow learner with poor scholastic performance and an intelligence quotient of 70-80. She attended a normal class, but struggled to pass class examinations every year. She has a proportionately short stature but not a coarse face. Her chin was small and supraorbital ridges hypoplastic with no ptosis. Her nose was long and alae nasi hypoplastic with overhanging columella. Her hair was thick and rough with some thinning on her scalp. She had increased hair on her back. Her fourth and fifth toes were short and all her finger nails were hypoplastic with thin and tapered fingers. Her fourth and fifth toes on both feet, and also the third toe on her right foot, were 
markedly hypoplastic. Clinodactyly was noted on the third, fourth and fifth toes on her right foot, and the third and fourth toes on her left foot. A skeletal survey did not show any radiographic bone abnormalities. Her bone age was 13-14 years and follicle-stimulating hormone was $1.57 \mathrm{IUl}^{-1}$ (normal range: $<5 \mathrm{IUl}^{-1}$ ). Ultrasonographic examination at 16 years (before menarche), showed a hypoplastic uterus and malrotation of both kidneys. No secondary sexual characteristics were recognized until she had menarche at 17 years. Now at age of 17 years, she is still short with a height of $141 \mathrm{~cm}(-5$ s.d.), weigh $31.3 \mathrm{~kg}$ ( -3 s.d.) and OFC $50.5 \mathrm{~cm}$ ( -4.5 s.d.). Clinical features are summarized in Supplementary Table 2.

Structural effects of SOX11 mutations. To determine the impact of the disease-causing mutations on human SOX11 structure and function, we mapped the mutation positions onto the crystal structure of mouse Sox $4^{9}$, that is analogous to human SOX11, and calculated free energy changes on the mutations using FoldX software ${ }^{10,11}$. The mutations lie in the highly conserved HMG domain, responsible for sequence-specific DNA binding (Fig. 2a) ${ }^{9}$. Ser60 is located in a helix of the HMG domain (Fig. 2a), therefore the S60P mutation may affect overall folding of the HMG domain and impair DNA binding of SOX11. FoldX calculations supported this prediction and the free energy change on the mutation was high enough to destabilize protein folding $\left(>10 \mathrm{kcal} \mathrm{mol}^{-1}\right) \quad$ (Fig. 2b) ${ }^{12}$. Conversely, Tyr116 forms a hydrophobic core with the side chains of DNA-recognition loops (Fig. 2a). The Y116C mutation has low free energy change $\left(<1 \mathrm{kcal} \mathrm{mol}^{-1}\right)$ (Fig. 2b), and is unlikely to significantly affect folding of the HMG domain, but instead may alter conformation of the DNA-recognition loop, which is important for DNA binding.

SOX11 mutations affect downstream transcription. Both mutations are located within the HMG domain, which is required for SOX11 transcriptional regulation of GDF5 (ref. 13). Luciferase assays using the GDF5 promoter in HeLa and ATDC5 cells, showed both mutant proteins had decreased transcriptional activities compared with wild type (WT) (Fig. 3).

SOX11 expression. SOX11 transcription levels were examined using multiple human complementary DNA (cDNA) panels. SOX11 was exclusively expressed in brain (foetus and adult) and heart (adult) tissues, supporting a role for SOX11 mutations in the brain features of CSS observed in the two patients (Supplementary Fig. 4; Supplementary Table 2).

In mice, targeted Sox11 disruption with a $\beta$-galactosidase marker gene results in $23 \%$ birth weight reduction and lethality after the first postnatal week in homozygotes, due to hypoplastic lungs and ventricular septation defects. In addition, skeletal malformations (including phalanges) and abdominal defects are observed $^{14}$. Physical and functional abnormalities in heterozygotes have not been described. However, in heterozygous mice, $\beta$-galactosidase expression revealed early ubiquitous expression throughout the embryo with upregulation in the central nervous system (CNS) and limb buds ${ }^{14}$.

sox11 knockdown experiments in zebrafish. We further investigated sox11 function in zebrafish. The zebrafish genome contains two orthologs of human SOX11, sox11a and sox11b, which are expressed in all cells until gastrulation and later become restricted to the developing $\mathrm{CNS}^{15,16}$. We knocked down zebrafish sox11a and sox11b (both single-exon genes) using translation-blocking morpholino oligonucleotides (MOs) (sox11a-MO, sox11b-MO and sox11a/b-MO), as previously described $^{17}$ (Supplementary Fig. 5a). Off-target effects of morpholino injections were excluded by repeated experiments, co-injecting with $t p 53 \mathrm{MO}$ or injecting into $t p 53^{z d f 1 / z d f 1}$ mutant fish ${ }^{18,19}$. sox $11 a$ - and sox11b-MO knockdown caused similar phenotypes, including smaller heads and body curvature (Supplementary Fig. 5b). Low-dose sox11a- (1.6 ng), sox11b$(1.6 \mathrm{ng})$ and sox11a/b- (1.6 ng) MO-injected embryos resulted in

a
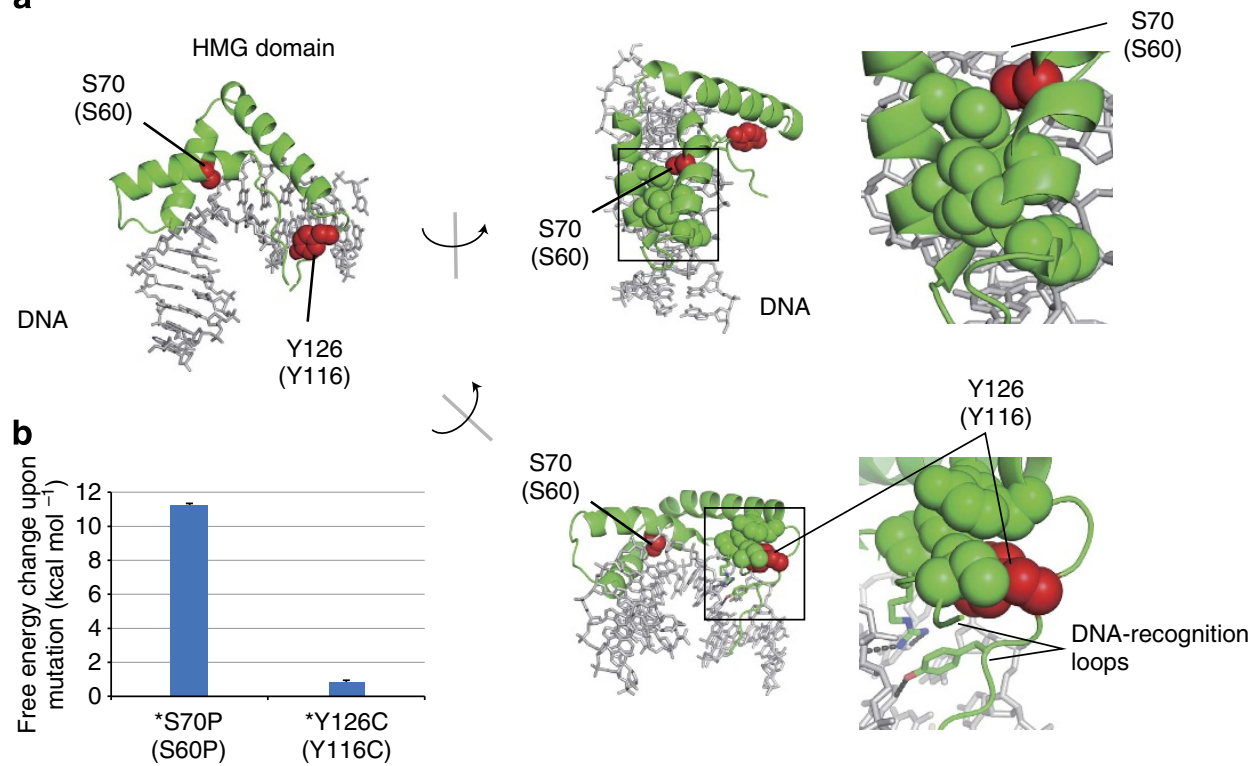

Figure 2 | Structural effects of Sox11 mutations. (a) Crystal structure of the mouse Sox4 HMG domain bound to DNA. Helices and loops are shown as green ribbons and threads, respectively. DNA is shown as grey sticks. Amino-acid residues at mutation sites are shown coloured red in the spacefilling model. In the middle and right images, some of the amino-acid residues involved in the hydrophobic core surrounding mutation points are shown coloured green in the space-filling model. Amino-acid numbering is indicated for mouse Sox4 with that for human SOX11 in parentheses. Hydrogen bonds are shown as black dotted lines. Molecular structures were drawn using PyMOL (http://www.pymol.org). (b) Free energy changes on the indicated mutations calculated by FoldX software. 

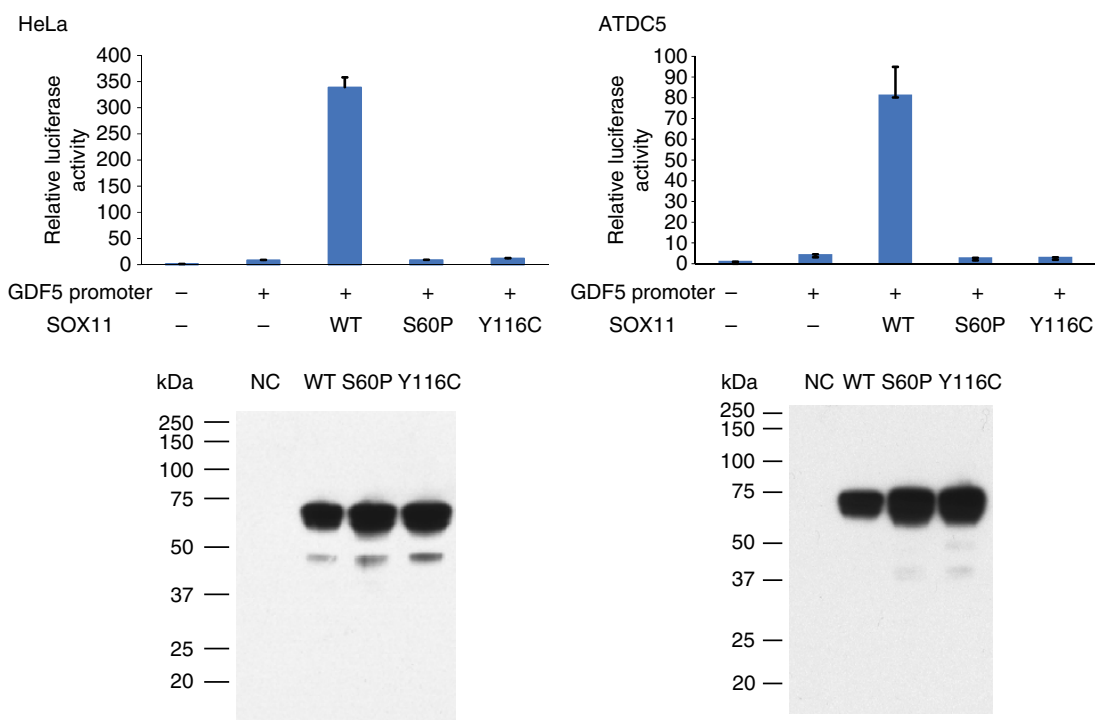

Figure 3 | SOX11 mutations affecting GDF5 promoter activity. Luciferase reporter assays measured transcriptional activity of the GDF5 promoter ( - 448/ + 319) (UCSC genome browser hg19: chr20: 34025709-34026457) in HeLa (left) and ATDC5 (right) cells. HeLa or ATDC5 cells were co-transfected with WT or mutant (S6OP and Y116C) SOX11 expression vector and reporter constructs containing either GDF5 promoter or empty vector (pGL3-basic). Relative luciferase activities compared with empty vector are presented as mean \pm s.d. for two independent experiments, with each experiment performed in triplicate (upper). Immunoblot analysis of transfected HeLa and ATDC5 cell extracts showing wild-type (WT) or mutant (S6OP and Y116C) SOX11 proteins (lower). Compared with WT, both SOX11 mutants reduced GDF5 transcriptional activities in HeLa and ATDC5 cells.

significant mortality (sox11a-MO, 49.3\%; sox $11 b-\mathrm{MO}$, $\sim 19.3 \%$; sox11a/b-MO, $\sim 53.0 \%$ ), compared with control-MO embryos ( 7.3\%) (Fig. 4a). Co-injection of WT human SOX11 mRNA (hSOX11-WT mRNA) with sox11a/b-MO improved morphant survival at $48 \mathrm{~h}$ post fertilization (hpf) $(25.5 \%$ lethality versus $49.3 \%$ lethality with sox11a/b-MO alone) $(P<0.01) \quad$ (Fig. 4a; Supplementary Fig. 5c). The affected phenotype of sox $11 \mathrm{a} / \mathrm{b}$ double morphants was partially rescued by hSOX11-WT mRNA overexpression (4.5\% normal for sox11a/ $b$-MO alone versus $36.5 \%$ for co-injection with hSOX11-WT mRNA and sox $11 a / b-\mathrm{MO}, P<0.01$ ) (Fig. 4a). In contrast, coinjection of either mutant hSOX11 mRNA (hSOX11-S60P and -Y116C mRNA) with sox11a/b-MO showed no significant rescue effects on lethal and affected phenotypes (Fig. 4a). There were significantly more normal phenotypes following hSOX11-WT mRNA and sox11a/b-MO co-injection, than with hSOX11mutant mRNA co-injections $(P<0.05)$. Head sizes in randomly selected embryos $(n \geq 10)$ of sox11a and sox11a/b morphants at $48 \mathrm{hpf}$ were significantly decreased $(P<0.05$ in both), but not significantly changed in sox $11 \mathrm{~b}$ morphant. Overexpression of hSOX11-WT mRNA restores sox11a/b double-morphant head size (in randomly selected embryos, $n \geq 10$ ), suggesting specific sox11 suppression by morpholino injection (Fig. 4b). Although the head size of hSOX11-mutant mRNA and sox11a/b-MOinjected embryos was slightly decreased, no significant difference was recognized between overexpression of hSOX11-WT or hSOX11-mutant mRNA and sox11a/b-MO co-injection (Fig. 4b).Staining with acridine orange and terminal deoxynucleotidyl TdT-mediated dUTP nick end labelling (TUNEL), found significant apoptotic increases exclusively in microcephalic embryos (Fig. 4c; Supplementary Fig. 6). Brain cell death was prevented by co-injection with hSOX11-WT mRNA, but not by mutant hSOX11 mRNAs (Fig. 4c). We also used $\mathrm{HuC} / \mathrm{D}$ (a marker for early postmitotic and mature neurons) and acetylated tubulin (an axonal marker) immunostaining at $48 \mathrm{hpf}$ to analyse neuronal cells in more detail (Supplementary Fig. 7). Decreased HuC/D-positive neurons, especially in the telencephalon and diencephalon, were observed in sox11 morphants (Supplementary Fig. 7a). The phenotype in sox11a/ $b$-MO-injected embryos was efficiently rescued by hSOX11-WT mRNA (Supplementary Fig. 7a). Reduction of HuC/D-positive neurons was unaltered by mutant hSOX11 mRNA overexpression and sox11a/b-MO injection (Supplementary Fig. 7a). Anti-acetylated tubulin staining also showed severely reduced axonal numbers in the forebrain, midbrain and hindbrain of sox11 morphants, compared with control-MO-injected embryos (Supplementary Fig. 7b). sox11a/b morphants showed phenotypic rescue when co-injected with hSOX11-WT mRNA, compared with mutant hSOX11 mRNAs (Supplementary Fig. 7b).

\section{Discussion}

We have identified SOX11 mutations in CSS. This is the first report of human mutations in SOXC (SOX4, SOX11 and $S O X 12)^{20}$. SOX11/sox 11 is required for neurogenesis, and loss of function in early embryos is sufficient to impair normal CNS development. Haploinsufficiency of other SOX genes (SOX2, SOX9 and SOX10) is known to cause human diseases ${ }^{21-23}$. It is interesting that mutations of SOX11 and other BAF subunit genes are mutually exclusive in CSS.

Sox11 was recently shown to form a transcriptional crossregulatory network downstream of the Pax6-BAF complex. The network drives neurogenesis and converts postnatal glia into neurons ${ }^{24}$. Brg1 (Smarca4) binds to the Sox11 promoter, and interaction with Pax6 is sufficient to induce Sox11 expression in neurosphere-derived cells in a Brg1-dependent manner ${ }^{24}$. Therefore, the Pax6-BAF complex activates a cross-regulatory transcriptional network, maintaining high expression of genes involved in neuronal differentiation and execution of cell lineage decisions $^{24}$. SOX11 mutations appear to be a rare cause of CSS as only 2 out of 92 patients (2.2\%) showed SOX11 abnormality and to be limited to the mild end of CSS phenotype. Abnormality of the upstream BAF complex tends to show a more severe phenotype compared with that of a downstream SOX11 mutation, which may indicate rather specific effects of SOX11 mutations on the CSS phenotype. 
a

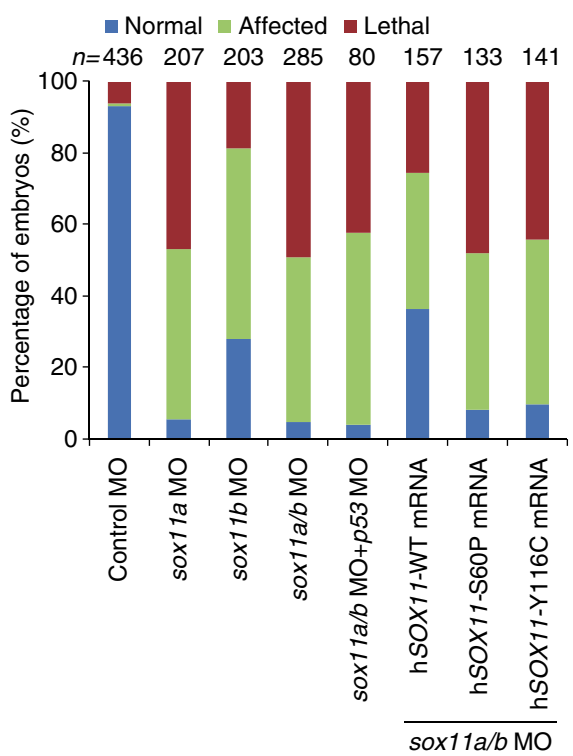

b

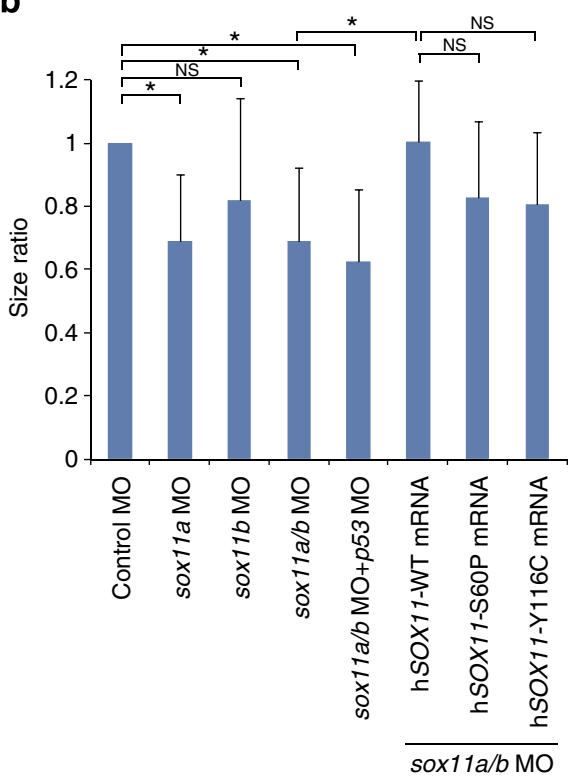

C

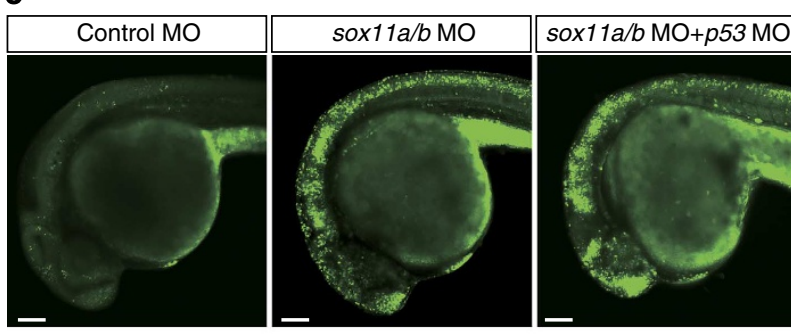

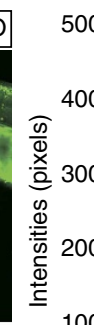
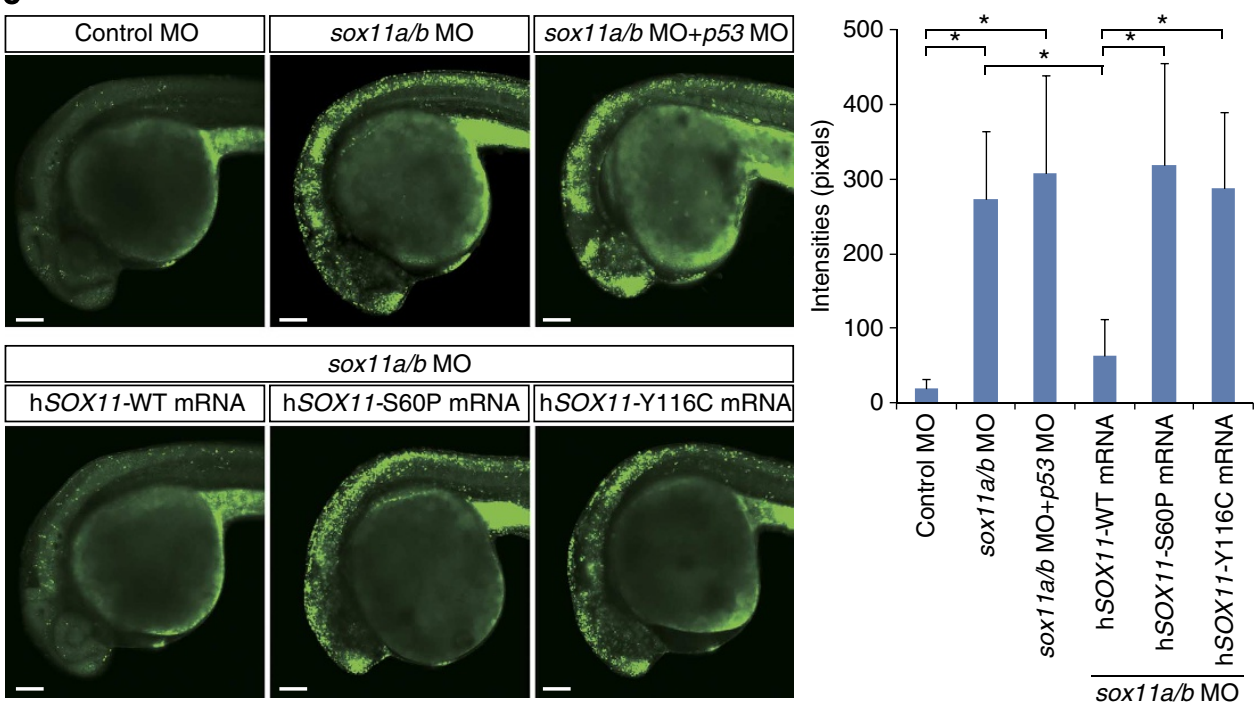

Figure 4 | sox11a/b knockdown experiments in zebrafish. (a) Embryos were injected with sox11-MO alone or with sox11- and tp53-MO or with sox11-MO and in vitro transcribed human SOX11 (hSOX11) mRNA (WT, wild type; S60P, p.Ser60Pro; Y116C, p.Tyr116Cys). Injected embryos were categorized as normal, affected and lethal at $48 \mathrm{hpf}$. The lethal and affected phenotype in sox11a/b-MO-injected embryos was partially rescued by WT hSOX11 mRNA overexpression. All experiments were performed more than twice and evaluated statistically with a Student's $t$-test. (b) Head size ratios of embryos with control-, sox11a-, sox11b- or sox11a/b-MO alone, or with sox11a/b- and tp53-MO or sox11a/b-MO and hSOX11 mRNA (WT or mutant) at 48 hpf ( $\mathrm{n} \geq 10$ ) (average of control-MO as 1). Dorsal views of midbrain width were measured. Data are represented as mean $\pm s$.d. ${ }^{\star} P<0.05$ by Student's $t$-test. NS, not significant. (c) Brain cell death in MO-injected embryos at $30 \mathrm{hpf}$ using acridine orange staining (lateral view). sox 11 morphants show increased cell death in the CNS. Scale bar, $100 \mu \mathrm{m}$. Quantification of acridine orange intensities in morphants are shown graphically (right, $n \geq 10$ ). Data are represented as mean \pm s.d. ${ }^{\star} P<0.001$ by Student's $t$-test.

In conclusion, mutations in both BAF complex genes and SOX11 result in the same phenotype (CSS), providing strong support for the BAF complex and SOX11 function in a common pathway, and play an important role in human brain development.

\section{Methods}

Subjects and clinical data. Patients were seen by their attending clinical geneticists. DNA samples were isolated from peripheral blood leukocytes using standard methods. Informed consent was obtained from the parents of the patients for experimental protocols and displaying participants' facial appearances in publications. This study was approved by the institutional review board of
Yokohama City University School of Medicine. A total of 92 patients were analysed, including 71 patients from a previous cohort and 21 new patients.

WES. Trio-based WES was performed in two families. Briefly, $3 \mu \mathrm{g}$ of genomic DNA was sheared using the Covaris $2 S$ system (Covaris, Woburn, MA) and partitioned using SureSelect Human All Exon V4 or V4 + UTRs (Agilent Technology, Santa Clara, CA), according to the manufacturer's instructions. Exon-enriched DNA libraries were sequenced using HiSeq2000 (Illumina, San Diego, CA) with 101-bp paired-end reads and 7-bp index reads. Four samples ( $2.5 \mathrm{pM}$ each, with different indexes) were run in one lane. Image analysis and base calling were performed using HiSeq Control Software/Real-Time Analysis and CASAVA1.8.2 (Illumina). Mapping to human genome hg19 was performed using Novoalign (http://www.novocraft.com/main/page.php?s=novoalign). Aligned reads were 
processed by Picard (http://picard.sourceforge.net) to remove PCR duplicates. Variants were called using the Genome Analysis Toolkit 1.5-21 (GATK v3) with best practice variant detection (http://gatkforums.broadinstitute.org/discussion/15/ best-practice-variant-detection-with-the-gatk-vl-x-retired), and annotated by Annovar (23 February 2012) (http://www.openbioinformatics.org/annovar/). Common variants registered in dbSNP137 (MAF $\geq 0.01)$ (http://genome.ucsc.edu/ cgi-bin/hgTrackUi?hgsid=281702941\&c=chr1\&g=snp137Flagged) were removed.

Prioritization of variants. From all the variants within exons and \pm 2 bp of intronic regions from exon-intron boundaries, those registered in either dbSNP137, 1,000 Genomes (http://www.1000genomes.org/), ESP 6500 (http:// evs.gs.washington.edu/EVS/) or our in-house (exome data from 408 individuals) databases, and those located within segmental duplications, were removed and we focused on heterozygous non-synonymous and splice site variants, which were subsequently confirmed by Sanger sequencing. SOX11 mutations in LOVD, http:// www.LOVD.nl/SOX11.

Structural modelling and free energy calculations. The crystal structure of the mouse Sox4 HMG domain bound to DNA (Protein Data Bank code 3U2B) was selected by SWISS-MODEL server 5 (ref. 25) as the structure most resembling human SOX11. To examine the missense mutations, mutational free energy changes were calculated using FoldX software (version 3.0) ${ }^{10,11}$. Calculations were repeated three times, and resultant data presented as average values with s.d.

SOX11 expression analysis in human tissues. TaqMan quantitative real-time PCR was performed using cDNAs from adult (Human MTC Panel I, \#636742, Clontech Laboratories, Mountain View, CA) and foetus (Human Fetal MTC Panel, \#636747, Clontech Laboratories). Pre-designed TaqMan probes for human SOX11 (Hs00167060_ml, Life Technologies Co., Carlsbad, CA) and human beta-actin (ACTB, 4326315E, Life Technologies Co.) were used. PCR was performed on a Rotor-Gene Q (QIAGEN, Valencia, CA) and expression levels normalized to $A C T B$, an internal standard gene, according to the $2^{-\Delta \Delta C t}$ method. Kidney expression was used as the standard $(1 \times)$.

Expression vectors. The SOX11 open-reading frame clone was purchased from Promega (Tokyo, Japan) and SOX11 mutants (c.178T $>$ C; p.Ser60Pro and c.347A > G; p.Tyr116Cys) generated by site-directed mutagenesis with the KOD Plus-Mutagenesis Kit (TOYOBO, Osaka, Japan). WT and mutant SOX11 cDNAs were PCR amplified and cloned into the pEF6/V5-His B mammalian expression vector (Life Technologies) using the In-Fusion PCR Cloning Kit (Clontech Laboratories), and also into the p3xFLAG-CMV-14 mammalian expression vector (Sigma, St Louis, MO). The GDF5 promoter $5^{\prime}$-flanking sequence $(-448 /+319)$ was PCR amplified and cloned into the pGL3-basic vector (Promega). All constructs were verified by Sanger sequencing. Human SOX11 cDNA can be obtained from GenBank/EMBL/DDBJ nucleotide core database under the accession code AB028641.1.

Immunostaining. Mouse neuroblastoma 2A (Neuro-2A) cells were cultured in Dulbecco's modified Eagle's medium (DMEM)-high glucose GlutaMAX supplemented with $10 \%$ fetal bovine serum (FBS) and penicillin-streptomycin (Life Technologies Co.). Neuro-2A cells were plated into 24 -well plates, $24 \mathrm{~h}$ before transfection. Each expression construct (200 ng) was transfected into Neuro-2A cells using X-tremeGENE 9 DNA Transfection Reagent (Roche Diagnostics, Indianapolis, IN). Twenty-four hours after transfection, cells were fixed in $4 \%$ paraformaldehyde (PFA)/phosphate-buffered saline (PBS) for $15 \mathrm{~min}$ at room temperature, and permeabilized in $0.1 \%$ Triton X-100/PBS for $5 \mathrm{~min}$ at room temperature. C-terminal V5-6xHis-tagged SOX11 proteins were detected using a mouse anti-V5 primary antibody (1:200; Life Technologies Co.) and an Alexa Fluor 546 Goat Anti-Mouse IgG secondary antibody (1:1,000; Life Technologies Co.). Smears were mounted in Vectashield mounting medium with DAPI (Vector Lab., Burlingame, CA). Confocal images were acquired using a Fluoview FV1000-D microscope (Olympus, Tokyo, Japan).

Luciferase assay. HeLa cells were cultured in DMEM-high glucose supplemented with penicillin $\left(50 \mathrm{units}^{-1}\right)$, streptomycin $\left(50 \mu \mathrm{g} \mathrm{ml}^{-1}\right)$ and $10 \%$ FBS. ATDC5 cells were cultured in DMEM/Ham's F-12 (1:1) supplemented with the above antibiotics and 5\% FBS. Cells were plated in 24 -well plates, $24 \mathrm{~h}$ before transfection, and transfections performed using TransIT-LT1 (Takara, Ohtsu, Japan) with pGL3 reporter (500 ng per well), effector ( $250 \mathrm{ng}$ per well) and pRL-SV40 internal control ( $6 \mathrm{ng}$ per well) vectors. Twenty-four hours after transfection, cells were harvested and luciferase activities measured using the PicaGene Dual SeaPansy Luminescence Kit (TOYO B-Net, Tokyo, Japan). Production of WT and mutant SOX11 proteins was assessed by immunoblot analysis with monoclonal anti-FLAG M2 HRP antibody (1:3,000; Sigma), following the manufacturer's instructions.

Morpholino and mRNA microinjection. Antisense translation-blocking morpholinos (MOs) for sox11a-(5'-CGCTGTTGTCCGTTTGCTGCACCAT-3'), sox $11 b-\left(5^{\prime} \text {-CTGTGCTCCGTCTGCTGCACCATGT-3' }\right)^{17}$, tp53-(5'-GCGCCAT TGCTTTGCAAGAATTG- $\left.3^{\prime}\right)^{18}$ and standard control- $\left(5^{\prime}\right.$-CCTCTTACCTCAG TTACAATTTATA- $3^{\prime}$ ) MO were obtained from GeneTools (Philomath, OR) and injected (or co-injected) into one- to two-cell-stage embryos at a final concentration of 0.1 or $0.2 \mathrm{mM}$. In rescue assays, capped human SOX11 mRNAs transcribed in vitro from $\mathrm{pEF} 6 / \mathrm{V} 5$-His B constructs were prepared using the mMessage mMachine T7 ULTRA Transcription Kit (Ambion, Carlsbad, CA), following the manufacturer's instructions, and injected into one-cell-stage embryos. For each MO knockdown and rescue experiment, embryos from the same clutch were used as experimental subjects and controls. Approximately $1 \mu \mathrm{g}$ of capped RNA was injected per embryo. The experiment was authorized by the institutional committee of fish experiments in the National Research Institute of Fisheries Science.

Cell death detection. To detect apoptotic cells in live embryos, embryos at $30 \mathrm{hpf}$ were manually dechorionated and incubated in acridine orange $\left(2 \mu \mathrm{g} \mathrm{ml}^{-1}\right.$ in egg water) at $28^{\circ} \mathrm{C}$ for $1 \mathrm{~h}$. After washing with egg water six times for $10 \mathrm{~min}$ each, embryos were anaesthetized with tricaine, mounted in $2 \%$ methylcellulose and examined by confocal microscopy. Apoptotic cells were also examined by the TUNEL assay, as previously described ${ }^{26}$. Embryos at $30 \mathrm{hpf}$, were fixed overnight in $4 \%$ PFA with PBS at $4{ }^{\circ} \mathrm{C}$ and stored in $100 \%$ methanol at $-20^{\circ} \mathrm{C}$. Samples were incubated in $100 \%$ acetone at $-20^{\circ} \mathrm{C}$ for $20 \mathrm{~min}$. Following fixation, the embryos were rinsed three times with PBS containing 0.1\% Tween-20. Samples were then permeabilized by treatment with $0.5 \%$ Triton X-100 and $0.1 \%$ sodium citrate in PBS for $15 \mathrm{~min}$. Embryos were subjected to the TUNEL assay by using the ApopTag Red in situ Apoptosis Detection Kit (Merck KGaA Millipore, Darmstadt, Germany) according to the manufacture's instruction.

Detection and quantitation of visible and fluorescent images. All animals were photographed under the same conditions using a LSM510 confocal microscope (Carl Zeiss, Jena, Germany). In each animal, acridine orange-positive cells were quantitated using a selection tool in Adobe Photoshop, for a colour range chosen by green colour selection of regions showing visually positive acridine orange staining. For analysis of embryos, defined head regions were selected in each embryo. Following pixel selection, a fuzziness setting of 0 was used, and chosen pixel numbers calculated using the image histogram calculation.

Whole-mount immunohistochemistry. For $\mathrm{HuC} / \mathrm{D}$ staining, embryos at $48 \mathrm{hpf}$ were fixed in $4 \%$ PFA overnight at $4{ }^{\circ} \mathrm{C}$ and dehydrated in methanol at $-20^{\circ} \mathrm{C}$. For acetylated tubulin staining, embryos at $48 \mathrm{hpf}$ were fixed in Dent's fixative $(80 \%$ methanol and $20 \%$ dimethyl sulphoxide) overnight at $4{ }^{\circ} \mathrm{C}$. Embryos were permeabilized with proteinase $\mathrm{K}$ followed by postfixation with $4 \%$ PFA and washed with PBSTX (PBS containing 0.5\% Triton X-100). After treating with $4 \%$ normal goat serum (NGS) in PBSTX for $2 \mathrm{~h}$ at room temperature, embryos were incubated with mouse anti-HuC/D (1:500, A21271, Life Technologies Co.) or mouse antiacetylated tubulin (1:1,000, T7451, Sigma) antibodies in 4\% NGS/PBSTX overnight at $4{ }^{\circ} \mathrm{C}$. Embryos were washed five times with PBSTX for 10 min each and incubated with goat anti-mouse fluorescein isothiocyanate secondary antibody diluted in $2 \%$ NGS/PBSTX for $2 \mathrm{~h}$ at room temperature. After washing five times for 10 min each, embryos were mounted in $2 \%$ methylcellulose and examined using a Fluoview FV1000-D confocal microscope (Olympus).

\section{References}

1. Ronan, J. L., Wu, W. \& Crabtree, G. R. From neural development to cognition: unexpected roles for chromatin. Nat. Rev. Genet. 14, 347-359 (2013).

2. Tsurusaki, Y. et al. Mutations affecting components of the SWI/SNF complex cause Coffin-Siris syndrome. Nat. Genet. 44, 376-378 (2012).

3. Tsurusaki, Y. et al. Coffin-Siris syndrome is a SWI/SNF complex disorder. Clin. Genet. 85, 548-554 (2014).

4. Santen, G. W. E. et al. Mutations in SWI/SNF chromatin remodeling complex gene ARID1B cause Coffin-Siris syndrome. Nat. Genet. 44, 379-380 (2012).

5. Santen, G. W. E. et al. Coffin-Siris syndrome and the BAF complex: genotypephenotype study in 63 patients. Hum. Mutat. 34, 1519-1528 (2013).

6. Wieczorek, D. et al. A comprehensive molecular study on Coffin-Siris and Nicolaides-Baraitser syndromes identifies a broad molecular and clinical spectrum converging on altered chromatin remodeling. Hum. Mol. Genet. 22, 5121-5135 (2013).

7. Van Houdt, J. K. et al. Heterozygous missense mutations in SMARCA2 cause Nicolaides-Baraitser syndrome. Nat. Genet. 44, 445-449 (2012).

8. Kosho, T. et al. Clinical correlations of mutations affecting six components of the SWI/SNF complex: detailed description of 21 patients and a review of the literature. Am. J. Med. Genet. A 161, 1221-1237 (2013).

9. Jauch, R., Ng, C. K., Narasimhan, K. \& Kolatkar, P. R. The crystal structure of the Sox4 HMG domain-DNA complex suggests a mechanism for positional interdependence in DNA recognition. Biochem. J. 443, 39-47 (2012).

10. Guerois, R., Nielsen, J. E. \& Serrano, L. Predicting changes in the stability of proteins and protein complexes: a study of more than 1000 mutations. J. Mol. Biol. 320, 369-387 (2002). 
11. Schymkowitz, J. et al. The FoldX web server: an online force field. Nucleic Acids Res. 33, W382-W388 (2005).

12. Khan, S. \& Vihinen, M. Performance of protein stability predictors. Hum. Mutat. 31, 675-684 (2010).

13. Kan, A. et al. SOX11 contributes to the regulation of GDF5 in joint maintenance. BMC Dev. Biol. 13, 4 (2013).

14. Sock, E. et al. Gene targeting reveals a widespread role for the high-mobilitygroup transcription factor Sox11 in tissue remodeling. Mol. Cell. Biol. 24, 6635-6644 (2004).

15. Rimini, R. et al. Expression patterns of zebrafish sox11A, sox11B and sox 21 . Mech. Dev. 89, 167-171 (1999).

16. de Martino, S. et al. Expression of sox11 gene duplicates in zebrafish suggests the reciprocal loss of ancestral gene expression patterns in development. Dev. Dyn. 217, 279-292 (2000).

17. Gadi, J. et al. The transcription factor protein sox 11 enhances early osteoblast differentiation by facilitating proliferation and the survival of mesenchymal and osteoblast progenitors. J. Biol. Chem. 288, 25400-25413 (2013).

18. Robu, M. E. et al. p53 activation by knockdown technologies. PLoS Genet. 3, e78 (2007).

19. Berghmans, S. et al. tp53 mutant zebrafish develop malignant peripheral nerve sheath tumors. Proc. Natl Acad. Sci. USA 102, 407-412 (2005).

20. Sarkar, A. \& Hochedlinger, K. The sox family of transcription factors: versatile regulators of stem and progenitor cell fate. Cell Stem Cell 12, 15-30 (2013).

21. Fantes, J. et al. Mutations in SOX2 cause anophthalmia. Nat. Genet. 33, 461-463 (2003)

22. Pingault, V. et al. SOX10 mutations in patients with WaardenburgHirschsprung disease. Nat. Genet. 18, 171-173 (1998).

23. Wagner, T. et al. Autosomal sex reversal and campomelic dysplasia are caused by mutations in and around the SRY-related gene SOX9. Cell 79, 1111-1120 (1994).

24. Ninkovic, J. et al. The BAF complex interacts with Pax6 in adult neural progenitors to establish a neurogenic cross-regulatory transcriptional network. Cell Stem Cell 13, 403-418 (2013).

25. Kiefer, F., Arnold, K., Kunzli, M., Bordoli, L. \& Schwede, T. The SWISSMODEL Repository and associated resources. Nucleic Acids Res. 37, D387-D392 (2009).

26. Koshimizu, E. et al. Embryonic senescence and laminopathies in a progeroid zebrafish model. PLoS ONE 6, e17688 (2011).

\section{Acknowledgements}

We thank the individuals and their families for participation in this study. We also thank Nobuko Watanabe for her technical assistance. This work was supported by the Ministry of Health, Labour and Welfare of Japan; the Japan Society for the Promotion of Science (a Grant-in-Aid for Scientific Research (B), and a Grant-in-Aid for Scientific Research (A)); the Takeda Science Foundation; the fund for Creation of Innovation Centers for Advanced Interdisciplinary Research Areas Program in the Project for Developing Innovation Systems; the Strategic Research Program for Brain Sciences; and a Grant-inAid for Scientific Research on Innovative Areas (Transcription Cycle) from the Ministry of Education, Culture, Sports, Science and Technology of Japan. The Indian Council of Medical Research, New Delhi is also appreciated for funding support for the DNA banking facility.

\section{Author contributions}

Y.T. and N.Ma. designed and directed the study. Y.T., E.K. and N.Ma. wrote the manuscript. H.O. and S.P. collected samples and provided subjects' clinical information. N.O. evaluated clinical information. Y.T., T.S., S.M., M.N., H.S., S.W., K.-i.Y. and N.Mi performed exome and Sanger sequencing. E.K., S.Ima. and M.Y. performed zebrafish experiments. I.K. and S.Ike. performed luciferase assays. M.S. and K.O. performed crystal structural analysis. Y.T. and H.K. analysed protein localization.

\section{Additional information}

Accession codes: Exome sequence data for CSS patients have been deposited in the Human Genetic Variation Browser under the accession code HGV0000001 (http:// www.genome.med.kyoto-u.ac.jp/SnpDB/repository/HGV0000001.html). Access to this data is controlled by the Yokohama City University Data Access Committee.

Supplementary Information accompanies this paper at http://www.nature.com/ naturecommunications

Competing financial interests: The authors declare no competing financial interests.

Reprints and permission information is available online at http://npg.nature.com/ reprintsandpermissions/

How to cite this article: Tsurusaki, Y. et al. De novo SOX11 mutations cause Coffin-Siris syndrome. Nat. Commun. 5:4011 doi: 10.1038/ncomms5011 (2014). 